\title{
Uptake, translocation and metabolism of polybrominated diphenyl ethers (PBDEs) and polychlorinated biphenyls (PCBs) in maize (Zea mays L.)
}

\author{
Sen Wang, Shuzhen Zhang*, Honglin Huang, Moming Zhao, Jitao Lv \\ State Key Laboratory of Environmental Chemistry and Ecotoxicology, Research Center for Eco-Environmental Sciences, Chinese Academy of Sciences,
} P.O. Box 2871, Beijing 100085, China

\section{A R T I C L E I N F O}

\section{Article history:}

Received 1 March 2011

Received in revised form 1 July 2011

Accepted 6 July 2011

Available online 27 July 2011

\section{Keywords:}

PBDEs

PCBs

Plant uptake

Translocation

Dehalogenation metabolism

\begin{abstract}
A B S T R A C T
A hydroponic experiment was conducted in the present study to investigate and compare plant uptake, translocation and metabolism of polybrominated diphenyl ethers (PBDEs) of BDE-15, BDE-28 and BDE-47 and polychlorinated biphenyls (PCBs) of PCB-15, PCB-28 and PCB-47 in maize. Root concentrations of BDE-15, BDE-28 and BDE-47 were consistently higher than PCB-15, PCB-28 and PCB-47, respectively. A significantly positive correlation was found between $\log$ RCF (root concentration factor) and $\log K_{\mathrm{ow}}$ of these PBDEs and PCBs, suggesting a control role of their partitioning in plant uptake. The translocation factors (TFs, $\mathrm{C}_{\text {stem }} / \mathrm{C}_{\text {root }}$ ) of PBDEs were generally lower than those of PCBs of the same halogen-substitutions, demonstrating easier transport of PCBs than PBDEs. Metabolites mono-, di- and tri-BDEs and PCBs were detected, suggesting the existence of in vivo metabolism of PBDEs and PCBs in maize. Dehalogenation and rearrangement of halogen atoms were identified, and some similarities but also significant differences existed between the PBDEs and PCBs. PBDEs in maize were, in general, more susceptible to metabolism compared with PCBs of the same halogen-substitutions. This is the first comparative report on the uptake, translocation and metabolism of PBDEs and PCBs in plants.
\end{abstract}

(c) 2011 Elsevier Ltd. All rights reserved.

\section{Introduction}

Polychlorinated biphenyls (PCBs) were historically used as dielectric fluids, heat exchangers in transformers and capacitors, and have been banned since the 1970s due to their high bioaccumulation and toxicity (Zhao et al., 2010). Polybrominated diphenyl ethers (PBDEs) are widely used as new additive brominated flame retardants (de Wit, 2002). Due to bioaccumulation and potential toxicity, the products of penta- and octa-BDEs have been banned or voluntarily withdrawn from use in some regions of the world (California State Assembly, 2003; Directive EEC, 2003). Both PBDEs and PCBs were detected in various environmental matrices such as soil, water, air, sediments and biotic samples (UNEP, 2002; Hassanin et al., 2004; Hites, 2004; Shen et al., 2006). Therefore, they represent potential risks to human and animal health.

PBDEs and PCBs have similar chemical structure and physicalchemical properties. Estimation by quantitative structureproperty relationship (QSPR) approach has indicated that PBDEs generally have higher partition coefficients than the PCBs of the same halogen-substitutions (Puzyn et al., 2008). PBDEs should be less persistent than PCBs of the same halogen-substitutions due to the lower energy of the $\mathrm{Br}-\mathrm{C}$ bond than $\mathrm{Cl}-\mathrm{C}$ bond. Nevertheless,

\footnotetext{
* Corresponding author. Tel.: +86 10 62849683; fax: +86 1062923563 .

E-mail address: szzhang@rcees.ac.cn (S. Zhang).
}

there has been no direct experimental evidence to support this suggestion.

Plant uptake of organic chemicals is an important process when considering the risks associated with land contamination, the role of vegetation in the global cycling of persistent organic pollutants, and the potential of industrial discharges to contaminate the food chain (Collins et al., 2006). Previous studies have investigated the uptake and accumulation of some PBDEs and PCBs in plants (Mueller et al., 2006; Liu and Schnoor, 2008; Huang et al., 2010; Xu et al., 2010). It is to be expected that PBDEs and PCBs may show different plant uptake patterns, since they have different properties such as partition coefficient. However, there has been no attempt to compare the uptake and translocation of PBDEs and PCBs in plants. Furthermore, degradation of PBDEs and PCBs is an important concern. PBDEs and PCBs have been shown to break down into lower halogenated congeners by microbial (He et al., 2006; Robrock et al., 2008; Van Aken et al., 2010) and photochemical degradation (Eriksson et al., 2004; Izadifard et al., 2008; Shih and Wang, 2009). However, only a few in vivo studies so far have been performed on debromination of PBDEs or dechlorination of PCBs in plants with very limited congeners, including CB-77 and BDE-209 (Liu and Schnoor, 2008; Huang et al., 2010; Wang et al., 2011). It is unclear whether there is difference between debromination of PBDEs and dechlorination of PCBs.

To resolve these questions, a hydroponic experiment was conducted in the present study to investigate the uptake and 

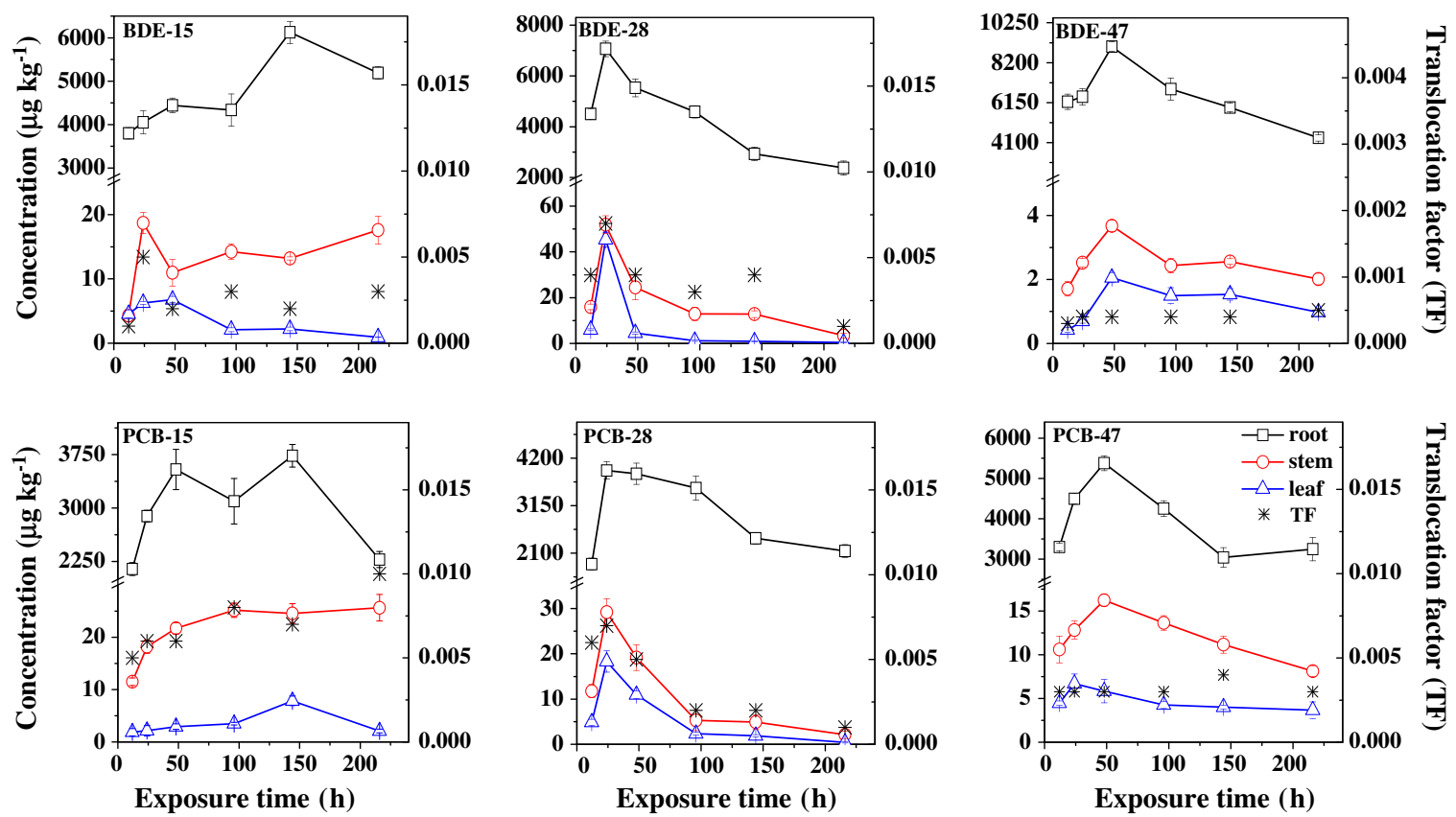

Fig. 1. Time-dependent of PBDE and PCB concentrations in maize, and their translocation factors (TFs). Error bars denote standard error of the mean.

translocation of PBDEs and PCBs in maize. BDE-15, BDE-28, BDE-47 and PCB-15, PCB-28, PCB-47 were selected as representatives of di-, tri- and tetra-BDEs as well as of PCBs, respectively. Concentrations of these PBDEs and PCBs as well as their dehalogenated metabolites in different parts of maize were analyzed and compared aiming to compare the uptake, translocation and metabolism of PBDEs and PCBs.

\section{Materials and methods}

\subsection{Chemicals}

The following standards were purchased from AccuStandard (AccuStandard, New Haven, USA): BDE-15, BDE-28 and BDE-47, PCB-15, PCB-28 and PCB-47, a standard solution of PBDEs containing 39 native congeners (mono- through hepta-BDEs), and a standard solution containing 39 PCB congeners (mono- through nona-PCBs). Standards of ${ }^{13} \mathrm{C}-\mathrm{PCB}-141$ and ${ }^{13} \mathrm{C}-\mathrm{PCB}-208$ were purchased from Cambridge Isotope Laboratory (Andover, MA, USA). All solvents used, i.e., hexane, dichloromethane and acetone, were of HPLC grade. Deionized water was used in all of the experiments. Anhydrous sodium sulfate $\left(\mathrm{Na}_{2} \mathrm{SO}_{4}\right)$, silica gel and alumina (100200 mesh) were washed with hexane and used after heating overnight at $150^{\circ} \mathrm{C}$.

\subsection{Exposure experiment}

Maize (Zea mays L.) was used as the test plant and the seeds were purchased from the Chinese Academy of Agricultural Sciences, Beijing, China. Seeds were sterilized by soaking in $3 \% \mathrm{H}_{2} \mathrm{O}_{2}$ solution for $30 \mathrm{~min}$, followed by thoroughly washing with deionized water and subsequently germinated on filter paper saturated with deionized water in the dark at $27^{\circ} \mathrm{C}$. Four days later, seedlings with $2-3 \mathrm{~cm}$ long were transplanted to containers containing half Hoagland nutrient solution. After $9 \mathrm{~d}$, maize plants were transferred to 150-mL glass-stoppered flask used as the exposure container, which was wrapped with aluminum foil to eliminate photolysis of PBDEs and PCBs. Autoclaved deionized water was used to prepare Hoagland nutrient solution, which was saturated

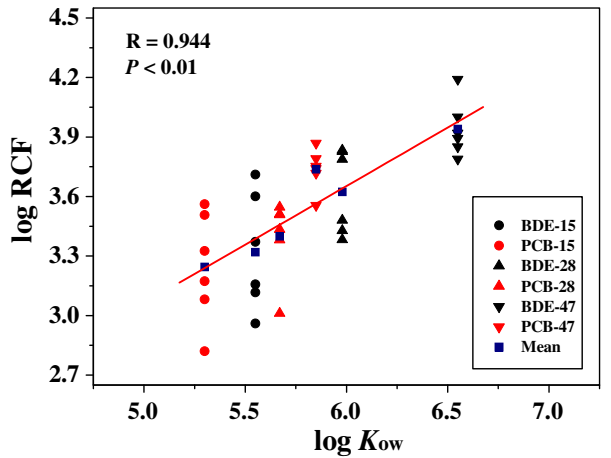

Fig. 2. Relationship between log RCF (values of all the exposures at different times) and $\log K_{\text {ow }}$ values of the PBDEs and PCBs.

Table 1

Dehalogenated metabolites of PBDEs and PCBs in maize. ${ }^{a}$

\begin{tabular}{ll}
\hline Congeners & Metabolites \\
\hline BDE-15 $\left(4,4^{\prime}-\right)$ & BDE-3 $(4-)$, BDE-2 (3-) \\
BDE-28 $\left(2,4,4^{\prime}-\right)$ & BDE-3 $(4-)$, BDE-2 $(3-)$, BDE- \\
& $15\left(4,4^{\prime}-\right)$, BDE-12, BDE-13 \\
& $\left(3,4-; 3,4^{\prime}-\right)$, BDE-37 \\
& $\left(3,4,4^{\prime}-\right)$, BDE-32 $\left(2,4^{\prime}, 6-\right)$ \\
& BDE-3 $(4-)$, BDE-2 $(3-)$, BDE- \\
BDE-47 $\left(2,2^{\prime}, 4,4^{\prime}-\right)$ & $15\left(4,4^{\prime}-\right)$, BDE-12, BDE-13 \\
& $\left(3,4-; 3,4^{\prime}-\right)$, BDE-28 \\
& $\left(2,4,4^{\prime}-\right)$ \\
& PCB-3 $(4-)$, PCB-1 $(2-)$ \\
PCB-15 $\left(4,4^{\prime}-\right)$ & PCB-8 $\left(2,4^{\prime}-\right)$, PCB-4 $\left(2,2^{\prime}-\right)$, \\
PCB-28 $\left(2,4,4^{\prime}-\right)$ & PCB-19 $\left(2,2^{\prime}, 6-\right)$, PCB-16 \\
& $\left(2,2^{\prime}, 3-\right)$ \\
PCB-47 $\left(2,2^{\prime}, 4,4^{\prime}-\right)$ & PCB-8 $\left(2,4^{\prime}-\right)$, PCB-15 $\left(4,4^{\prime}-\right)$, \\
& PCB-28 $\left(2,4,4^{\prime}-\right)$, PCB-19 \\
& $\left(2,2^{\prime}, 6-\right)$, PCB-16 $\left(2,2^{\prime}, 3-\right)$, \\
& PCB-18 $\left(2,2^{\prime}, 5-\right)$, PCB-25 \\
& $\left(2,3^{\prime}, 4-\right)$
\end{tabular}

${ }^{a}$ Data within parenthesis are halogen substitutions on biphenyl structure. 
by oxygen to reduce the metabolism of anaerobic microorganisms. Standard solutions of BDE-15, BDE-28, BDE-47 and PCB-15, PCB28 , PCB-47, dissolved in acetone, were added to make exposure solution with their concentrations at 19.5, 21.6, 15.5, 19.8, 20.7, and $16.8 \mu \mathrm{g} \mathrm{L}^{-1}$, respectively, aiming to set the initial concentrations lower than their water solubility. All these were conducted in a laminar flow hood.

The containers were positioned randomly and re-randomized every day. The photoperiod was set $14 \mathrm{~h} \mathrm{~d}^{-1}$ at a light intensity of $250 \mu \mathrm{mol} \mathrm{m} \mathrm{m}^{-2} \mathrm{~s}^{-1}$ provided by supplementary illumination. The day/night temperature regime was $25^{\circ} \mathrm{C} / 20^{\circ} \mathrm{C}$ and the relative humidity was maintained $60-70 \%$. Approximately $10 \mathrm{~mL} \mathrm{~d}^{-1}$ of autoclaved deionized water saturated with oxygen was injected into containers to compensate for the transpiration losses. Unplanted control and blank control (without PBDE and PCB exposure) were included as controls and all the treatments were set up in triplicate.

\subsection{Sample preparation}

Maize plants were harvested after intervals of $12,24,48,96$ 144 , and $216 \mathrm{~h}$. Root samples were first carefully rinsed with deionized water to remove any adhering PBDEs or PCBs, and rinses were collected and combined into the exposure solutions. Then maize leaves, stems and roots were rinsed thoroughly with deionized water, blotted with filter paper and weighed. All the samples were then frozen at $-50^{\circ} \mathrm{C}$ overnight, freeze-dried for $48 \mathrm{~h}$ in a lyophilizer (FD-1, Beijing Boyikang Instrument Ltd., Beijing, China), and weighed to determine their dry weight. The dried samples were chopped finely and stored in glass containers at $-20^{\circ} \mathrm{C}$ before chemical analysis.

\subsection{Extraction and analysis}

Extraction and cleanup of PBDEs and PCBs in solutions and plant samples were based on the method of López et al. (2009) and Wang et al. (2011), and the detail method was provided in the Supplementary material. ${ }^{13} \mathrm{C}$-PCB-141 was added as surrogate standard to the samples prior to extraction and ${ }^{13} \mathrm{C}-\mathrm{PCB}-208$ was added to the final solutions as an internal standard. An Agilent
7890 GC-MS (5975 inert) (Agilent, Palo Alto, CA, USA) and a HP5MS column $(30 \mathrm{~m} \times 0.32 \mathrm{~mm} \times 0.25 \mu \mathrm{m})$ (J \& W Scientific, Folsom, CA) were used for PBDEs and PCBs analysis. Quality assurance and quality control of the extraction and analysis are provided in the Supplementary material.

\subsection{Data analysis}

The data were subjected to statistical analysis by using the SPSS version 11.5 software package. Means and standard deviation were calculated for triplicates. Analysis of paired-samples $T$-test was used to examine the significance of PBDEs, PCBs and their dehalogenated metabolites accumulation in roots, stems and leaves at all exposure time.

\section{Results and discussion}

\subsection{Uptake of PBDEs and PCBs by maize root}

Fig. 1 shows time-dependent concentrations of the tested PBDEs and PCBs in maize. Concentrations of BDE-15, BDE-28, BDE-47 and PCB-15, PCB-28, PCB-47 increased with the increasing exposure time until $144,24,48 \mathrm{~h}$, respectively, and then decreased. In comparison, root concentrations of BDE-15, BDE-28 and BDE-47 were consistently higher than those of PCB-15, PCB-28 and PCB-47, respectively. To compare the uptake abilities of PBDEs and PCBs by roots, root concentration factor (RCF), defined as the ratio of concentration in roots $\left(\mu \mathrm{g} \mathrm{kg}^{-1}\right)$ to concentration in external solution ( $\mu \mathrm{g} \mathrm{L}^{-1}$ ), was calculated (Trapp, 2000). The RCFs for the PBDEs and PCBs were plotted against their $\log K_{\text {ow }}$ values and shown in Fig. 2. The mean RCF values were in the order: PCB-15 < BDE$15<$ PCB-28 < BDE-28 < PCB-47 < BDE-47. Similar observation of higher bioconcentration factors for BDEs (BDE-47, BDE-99 and BDE-153) than PCBs with a similar number of chlorines was also reported previously (Tomy et al., 2004). Furthermore, a significantly positive linear relationship was found between $\log$ RCF and $\log K_{\text {ow }}$ of PBDEs and PCBs all together (Fig. 2; $R=0.944$, $P<0.01$ ), demonstrating that partitioning of the PBDEs and PCBs has a key role in controlling their plant uptake.
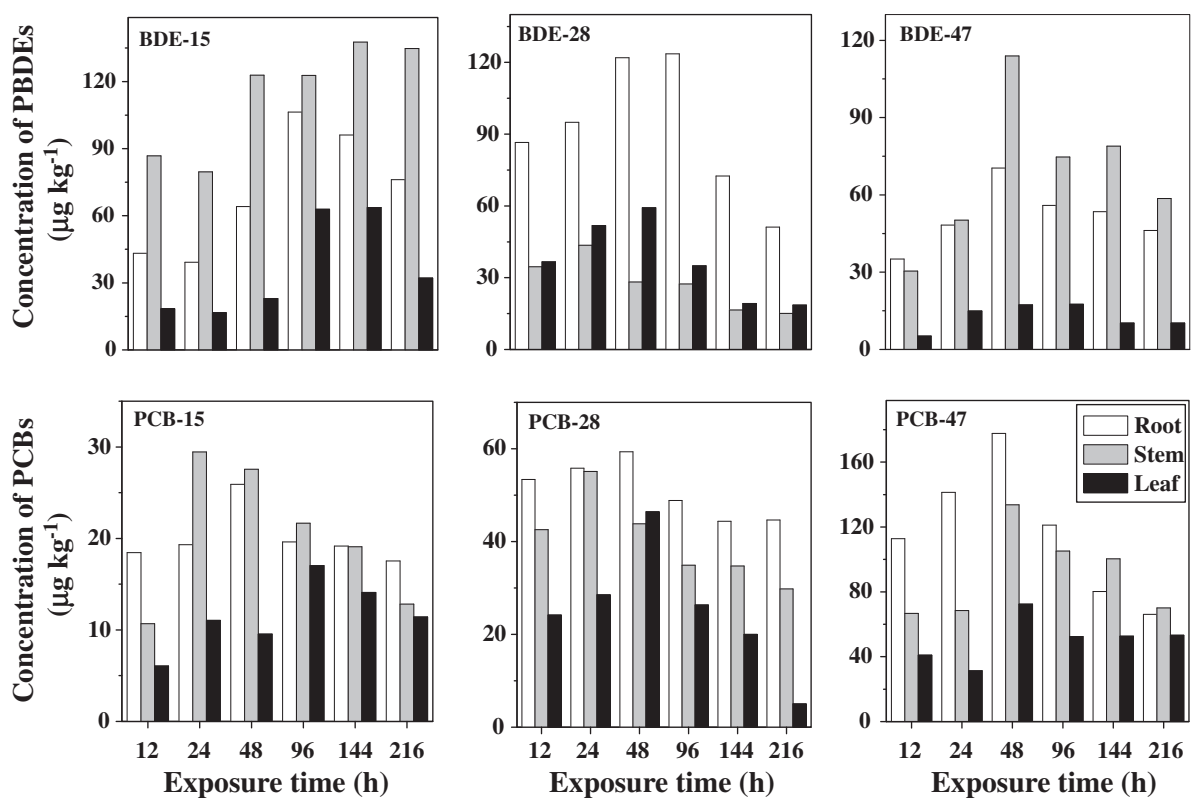

Fig. 3. Time-dependent of the total debrominated PBDE and dechlorinated PCB concentrations in maize. 

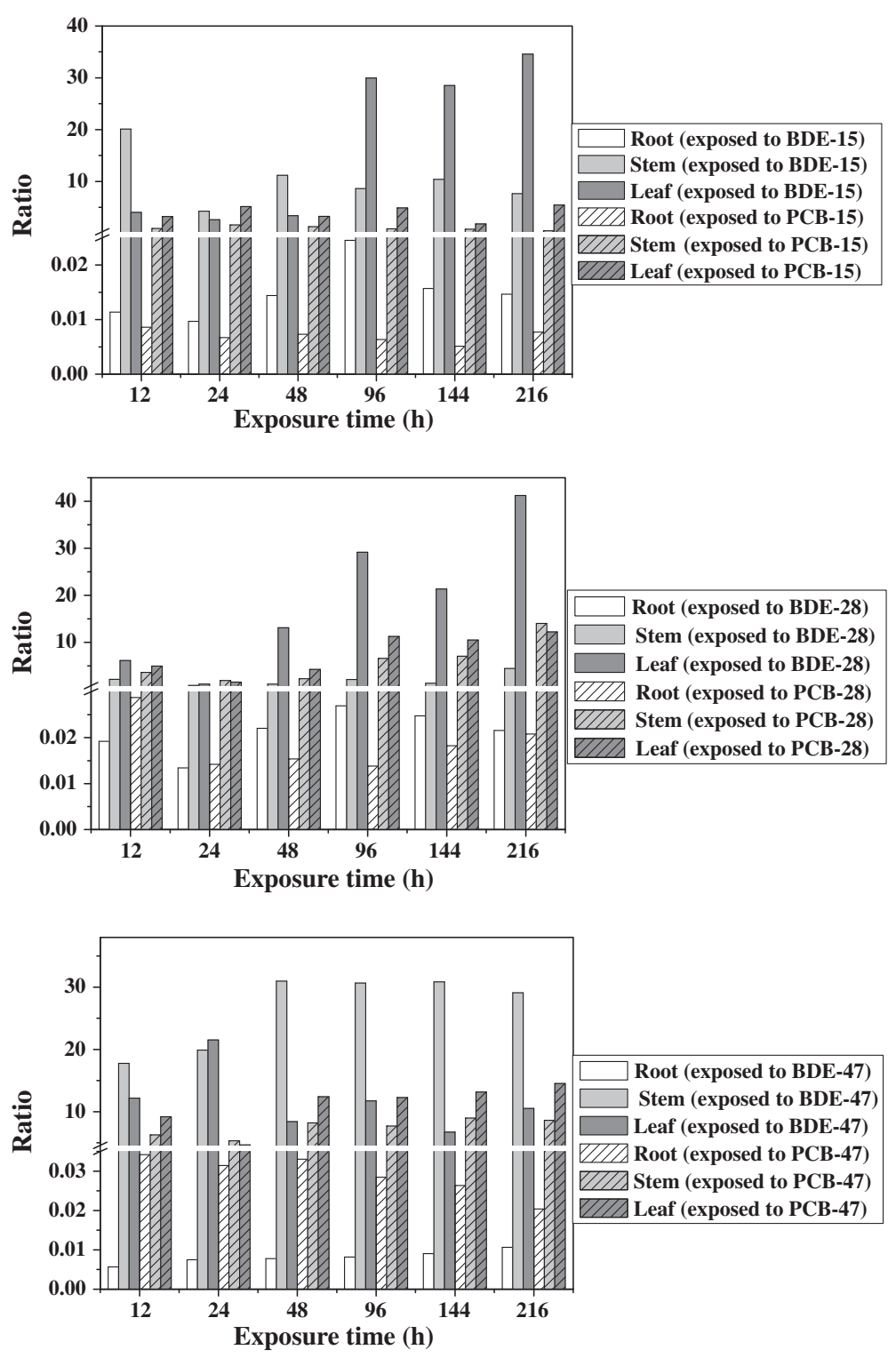

Fig. 4. Ratios of the total dehalogenated metabolites to the parent congeners in maize exposed to PBDEs and PCBs, respectively.

\subsection{Translocation of PBDEs and PCBs within maize}

Accumulation of PBDEs and PCBs in plants may result from a combination of acropetal translocation after root uptake and foliar uptake from the air. Concentrations of PBDEs and PCBs in the control plants without contamination exposure are shown in Table S2, Supplementary material. Only BDE-15, BDE-47 and PCB-47 were detected in very limited stem and leaf samples at concentrations of $0.52-1.25,0.33-0.35,0.20-0.24 \mu \mathrm{g} \mathrm{kg}^{-1}$, respectively, which accounted for less than $0.1 \%$ of the total PBDEs or PCBs in the exposed plants. This implies that there was no appreciable contribution from foliar uptake to the accumulation of these compounds in the aboveground parts of maize in this experiment. Furthermore, PBDEs and PCBs were detected in all the maize stem and leaf samples with the concentrations following the order: roots $\gg$ stem$\mathrm{s}>$ leaves. These results suggest the acropetal translocation of PBDEs and PCBs in maize.

Concentrations of BDE-28, BDE-47 and PCB-28, PCB-47 in stems and leaves increased with the increasing exposure time until 24 , $48 \mathrm{~h}$ and then decreased, following much the same pattern as the root concentrations. Similarly, concentrations of BDE-15 and PCB15 in leaves also increased with increasing exposure time until 48 and $144 \mathrm{~h}$, respectively, and then decreased; whereas their concentrations in the stems increased consistently over the exposure time. Translocation factors (TFs, $\mathrm{C}_{\text {stem }} / \mathrm{C}_{\text {root }}$ ) of PBDEs and PCBs were calculated and plotted in Fig. 1 for the further analysis of acropetal translocation. It is difficult to make a brief comparison of the TF values among different PBDE and PCB congeners or between PBDE and PCB of the same halogen-substitutions when the data for different exposure time were taken into consideration. Nevertheless, the TF values of PCB-15 and PCB-47 were higher than those of BDE-15 and BDE-47, respectively, suggesting easier transport of PCB-15 and PCB-47 than BDE-15 and BDE-47. But no obvious difference was found between the TF values of PCB-28 and BDE-28. Furthermore, BDE-47 had much lower TFs than all the other congeners. In comparison, translocation of PBDEs and PCBs in plants was more complicated than their root uptake which is mainly determined by their partitioning. Metabolism and acropetal translocation of PBDEs and PCBs inside plants would contribute much uncertainty of the TFs. 


\subsection{Dehalogenated metabolites of PBDEs and PCBs in maize}

Dehalogenated metabolites of mono-, di- and tri-BDEs and PCBs were detected in maize root, stem and leaf samples. The dehalogenation products are summarized in Table 1 and the concentrations are provided in Table S4-5, Supplementary material. No dehalogenated metabolites were measured in the hydroponic solutions with the exception of BDE-28 exposure experiment in which BDE-2, BDE-12, BDE-13, BDE-15, BDE-32 and BDE-37 were detected in the solutions before plant exposure. But they all together accounted for less than $0.8 \%$ of BDE- 28 added, and were much lower than their concentrations in the exposed maize (Table S3, Supplementary material). These results suggest the occurrence of dehalogenation of the PBDEs and PCBs in maize. Dehalogenated metabolites were detected in maize after only a $12 \mathrm{~h}$ exposure, indicating metabolism occurred very rapidly.

Time-dependent accumulations of dehalogenated metabolites in maize are shown in Fig. 3 and S1-2, Supplementary material (Fig. 3 as the total concentrations and Fig. S1-2 as the bromine or chlorine number). Accumulations of the dehalogenated metabolites in each part of maize generally increased first and then decreased at different degree for different congeners. The decrease in accumulation may be caused by the further metabolism to other lower dehalogenated congeners or hydroxylated and methoxylated analogues.

In comparison, the total concentrations of the dehalogenated metabolites were relatively lower in leaves than in roots and stems with an exception of higher concentrations in leaves than in stems of BDE-28 exposed maize. Differences between the total concentrations of the dehalogenated metabolites in roots and stems were not significant $(P>0.05)$ for the exposures of PCB-15 and PCB-47 and BDE-47. Whereas debrominated metabolites were significant higher in stems than in roots $(P<0.05)$ of BDE-15 exposed maize, and opposite results were obtained with the BDE-28 and PCB-28 exposure tests. Considering the distinct accumulation difference of the parent congeners in leaves, stems and roots, as well as the mass difference of each plant part, ratios of the total dehalogenated metabolites to the parent congeners in each part of maize were further calculated and depicted in Fig. 4. The ratios were in the decreasing order of roots $\ll$ stems $<$ leaves for all the exposed
PCB congeners. Similar trend was also obtained for the exposures of BDE-15 and BDE-28 with the exceptions of the results for the early exposure to BDE-15 (the first $48 \mathrm{~h}$ ). However, for BDE-47 exposure treatment, the ratios for stems were consistently higher than those for leaves. A detailed interpretation of dehalogenation in plants is difficult since accumulation of dehalogenated metabolites in maize is attributed to a combination of dehalogenation and translocation of the dehalogenated metabolites inside plants. Nevertheless, it is still safe to get a general conclusion based on the above results that these PBDEs and PCBs are more susceptible to metabolism in maize leaves and stems compared with roots. In comparison, the total concentrations of dehalogenated metabolites and their ratios to the parent congeners were lower for PCB-15 and PCB-28 in all parts of maize than the respective data for BDE-15 and BDE-28 treatments with only exception of the results for PCB-28 and BDE-28 in maize stems, in agreement with our speculation that PBDEs were less persistent than PCBs of the same halogen-substitutions due to the lower energy of the $\mathrm{Br}-\mathrm{C}$ bond than $\mathrm{Cl}-\mathrm{C}$ bond, and therefore debrominations of BDE-15 and BDE-28 were more prevalent than dechlorinations of PCB-15 and PCB-28. However, dechlorinated metabolites in PCB-47 exposed maize were higher than debrominated metabolites of BDE-47, and the ratios of the dechlorinated metabolites to PCB-47 in maize roots and leaves were also higher than the data obtained for the BDE-47 exposure, probably because of a stronger partitioning of BDE-47 compared with PCB-47 into plant organic components due to its higher $\log K_{\text {ow }}$ value reduced its dehalogenation.

\subsection{Dehalogenation and halogen rearrangement of PBDEs and PCBs in maize}

Based on the dehalogenated metabolites detected, debromination and rearrangement of bromine atoms of PBDEs or dechlorination and rearrangement of chlorine atoms of PCBs were assumed to occur in maize (Fig. 5 and S3, Supplementary material). Taking the BDE-47 and PCB-47 exposures as examples, BDE-3, BDE-15 and $\mathrm{BDE}-28$ detected were presumed to be the products of the debromination of BDE-47, and the bromine atom rearrangement of BDE-47 might form BDE-2, BDE-12 and BDE-13 during metabolism

(A)

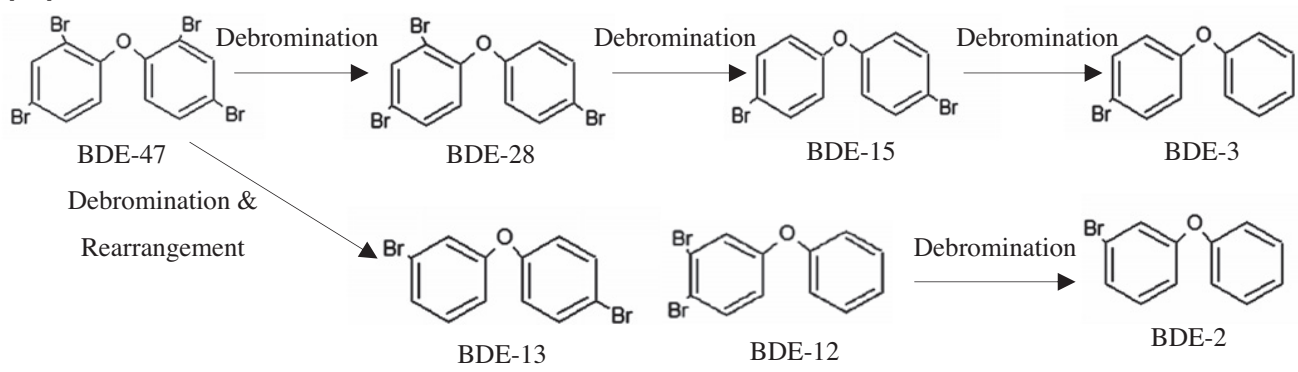

(B)
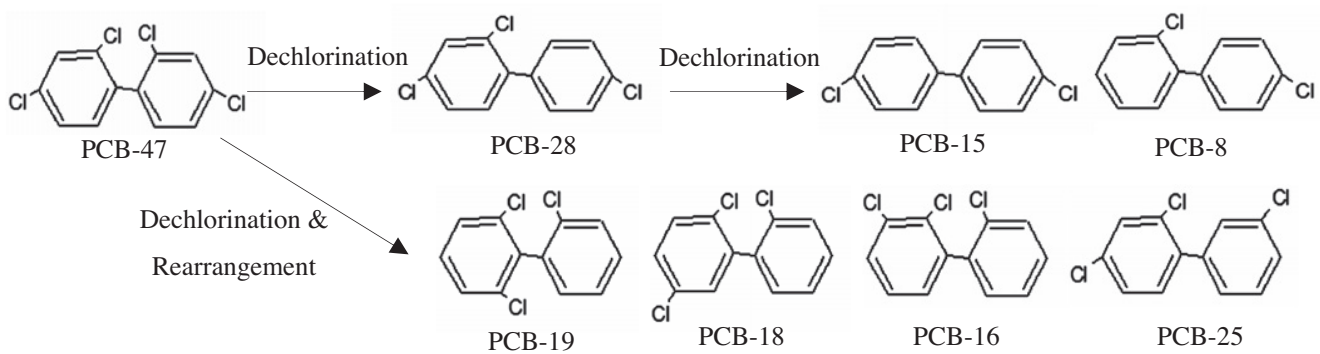

Fig. 5. Metabolism pathways of BDE-47 (A) and PCB-47 (B) in maize. 
by maize; whereas $\mathrm{PCB}-8, \mathrm{PCB}-15$ and $\mathrm{PCB}-28$ were likely the dechlorinated products of $\mathrm{PCB}-47$, and the chlorine atom rearrangement of PCB-47 formed PCB-19, PCB-18, PCB-16 and PCB-25 (Fig. 5). Dehalogenation of BDE-47 and PCB-47 had some similarities but also significant differences. Dechlorination and rearrangement reactions have been observed in PCB-77 exposed poplars and switchgrass in a previous study (Liu et al., 2009). For the dehalogenated metabolism of PBDEs and PCBs, some studies have shown that chlorine and bromine atoms are preferentially removed from the meta- and para-positions on the biphenyl structure by microbial degradation (Gerecke et al., 2005; Field and Sierra-Alvarez, 2008). On the other hand, some reports have demonstrated that the chlorine and bromine atoms at orthopositions show much higher elimination efficiency than those at meta- and para-positions (Miao et al., 1999; Fang et al., 2008). In this study, all the PBDEs and PCBs investigated were at the orthoor para-positions. For BDE-28 and BDE-47 exposures, the metabolites of the debromination at para-positions (BDE-8 and BDE-17) were not detected since the bromine atoms at the ortho-positions might depart more easily to form BDE-15 and BDE-28. For PCB-47 exposure, the metabolites of the dechlorination at orthopositions (PCB-15 and PCB-28) and ortho- and para-positions (PCB-8) were both detected. Relatively higher concentrations of PCB-15 and PCB-28 were detected than the other congeners in PCB-47 exposed maize (Table S5, Supplementary material), suggesting that the ortho-chlorines depart more easily to form PCB15 and PCB-28 compared with the para-chlorines. But for PCB-28 exposure, no metabolite of dechlorination at ortho-positions (PCB-15) was detected. The chlorine atom at the para-positions might depart more easily to form PCB-8 compared with the ortho-positions.

Relatively higher concentrations of mono-BDEs than di- and tri-BDEs were detected in BDE-28 and BDE-47 exposed maize (Fig. S1, Supplementary material), possibly attributing to the further debromination of di- and tri-BDEs within maize. Oppositely, no mono-PCBs were detected in maize exposed to PCB-28 and PCB47. This comparison further demonstrates that the debrominated metabolism of PBDEs in maize is stronger than dechlorinated metabolism of PCBs of the same halogen-substitutions.

\section{Conclusions}

This study demonstrates distinct differences of plant uptake, translocation and metabolism between BDE-15, BDE-28, BDE-47 and PCB-15, PCB-28, PCB-47. The differences can be summarized as follows: (1) PBDEs showed higher accumulation in maize roots than PCBs of the same halogen-substitutions due to the higher partition coefficients $\left(\log K_{\mathrm{ow}}\right.$ ) of the PBDEs than those of the PCBs. (2) Higher TF values of PCB-15 and PCB-47 than those of BDE-15 and BDE-47 suggest the easier transport of these PCBs than PBDEs of the same halogen-substitutions. However, no obvious difference was found between the TF values of PCB-28 and BDE-28. (3) Dehalogenation and rearrangement of bromine atoms of PBDEs or chlorine atoms of PCBs existed in maize. PBDEs were, in general, more susceptible to metabolism compared with PCBs of the same halogen-substitutions in maize. This comparative study is of great significance in predicting the fate of PBDEs and PCBs in the environment.

\section{Acknowledgements}

This work was funded by the National Natural Science Foundation of China (Projects 40730740, 20921063 and 20737003) and the National Basic Research Program of China (Project 2009CB421603).

\section{Appendix A. Supplementary material}

Supplementary data associated with this article can be found, in the online version, at doi:10.1016/j.chemosphere.2011.07.002.

\section{References}

California State Assembly, 2003. Assembly Bill No. 302. California State Assembly, Sacramento, CA.

Collins, C., Fryer, M., Grosso, A., 2006. Plant uptake of non-ionic organic chemicals. Environ. Sci. Technol. 40, 45-52.

De Wit, C.A., 2002. An overview of brominated flame retardants in the environment. Chemosphere 46, 583-624.

EEC, 2003. Directive 2003/11/EC of the european parliament and of the council of 6 february 2003 amending for the 24th time council directive 76/769/EEC relating to restrictions on the marketing and use of certain dangerous substances and preparations (pentabromodiphenyl ether and octabromodiphenyl ether) Official J. Eur. Union L42, 45-46.

Eriksson, J., Green, N., Marsh, G., Bergman, Å., 2004. Photochemical decomposition of 15 polybrominated diphenyl ether congeners in methanol water. Environ. Sci. Technol. 38, 3119-3125.

Fang, L., Huang, J., Yu, G., Wang, L.N., 2008. Photochemical degradation of six polybrominated diphenyl ether congeners under ultraviolet irradiation in hexane. Chemosphere 71, 258-267.

Field, J.A., Sierra-Alvarez, R., 2008. Microbial transformation and degradation of polychlorinated biphenyls. Environ. Pollut. 155, 1-12.

Gerecke, A.C., Hartmann, P.C., Heeb, N.V., Kohler, H.P.E., Giger, W., Schimid, P., Zennegg, M., Kohler, M., 2005. Anaerobic degradation of decabromodiphenyl ether. Environ. Sci. Technol. 39, 1078-1083.

Hassanin, A., Breivik, K., Meijer, S.N., Steinnes, E., Thomas, G.O., Jones, K.C., 2004 PBDEs in European background soils: levels and factors controlling their distribution. Environ. Sci. Technol. 38, 738-745.

He, J.Z., Robrock, K.R., Alvarez-Cohen, L., 2006. Microbial reductive debromination of polybrominated diphenyl ethers. Environ. Sci. Technol. 40, 4429-4434.

Hites, R.A., 2004. Polybrominated diphenyl ethers in the environment and in people: a meta-analysis of concentrations. Environ. Sci. Technol. 38, 945956.

Huang, H.L., Zhang, S.Z., Christie, P., Wang, S., Xie, M., 2010. Behavior of decabromodiphenyl ether (BDE-209) in the soil plant system: uptake, translocation, and metabolism in plants and dissipation in soil. Environ. Sci. Technol. 44, 663-670.

Izadifard, M., Achari, G., Langford, C.H., 2008. The pathway of dechlorination of PCB congener by a photochemical chain process in 2-propanol: the role of medium and quenching. Chemosphere 73, 1328-1334.

Liu, J.Y., Schnoor, J.L., 2008. Uptake and translocation of lesser-chlorinated polychlorinated biphenyls (PCBs) in whole hybrid poplar plants after hydroponic exposure. Chemosphere 73, 1608-1616.

Liu, J.Y., Hu, D.F., Jiang, G.B., Schnoor, J.L., 2009. In vivo biotransformation of $3,3^{\prime}, 4,4^{\prime}$-tetrachlorobiphenyl by whole plants-poplars and switchgrass. Environ. Sci. Technol. 43, 7503-7509.

López, P., Brandsma, S.A., Leonards, P.E.G., De Boer, J., 2009. Methods for the determination of phenolic brominated flame retardants, and by-products, formulation intermediates and decomposition products of brominated flame retardants in water. J. Chromatogr. A 1216, 334-345.

Miao, X.S., Chu, S.G., Xu, X.B., 1999. Degradation pathways of PCBs upon UV irradiation in hexane. Chemosphere 39, 1639-1650.

Mueller, K.E., Mueller-Spitz, S.R., Henry, H.F., Vonderheide, A.P., Soman, R.S., Kinkle, B.K., Shann, J.R., 2006. Fate of pentabrominated diphenyl ethers in soil: abiotic sorption, plant uptake, and the impact of interspecific plant interactions. Environ. Sci. Technol. 40, 6662-6667.

Puzyn, T., Suzuki, N., Haranczyk, M., 2008. How do the partitioning properties of polyhalogenated POPs change when chlorine is replaced with bromine. Environ. Sci. Technol. 42, 5189-5195.

Robrock, K.R., Korytar, P., Alvarez-Cohen, L., 2008. Pathways for the anaerobic microbial debromination of polybrominated diphenyl ethers. Environ. Sci. Technol. 42, 2845-2852.

Shen, L., Wania, F., Lei, Y.D., Teixeira, C., Muir, D.C.G., Xiao, H., 2006. Polychlorinated biphenyls and polybrominated diphenyl ethers in the North American atmosphere. Environ. Pollut. 144, 434-444.

Shih, Y.H., Wang, C.K., 2009. Photolytic degradation of polybromodiphenyl ethers under UV-lamp and solar irradiations. J. Hazard. Mater. 165, 34-38.

Tomy, G.T., Palace, V.P., Halldorson, T., Braekevelt, E., Danell, R., Wautier, K., Evans, B., Brinkworth, L., Fisk, A.T., 2004. Bioaccumulation, biotransformation, and biochemical effects of brominated diphenyl ethers in juvenile lake trout (Salvelinus namaycush). Environ. Sci. Technol. 38, 1496-1504.

Trapp, S., 2000. Modelling uptake into roots and subsequent translocation of neutral and ionisable organic compounds. Pest Manage. Sci. 56, 767-778.

UNEP, United Nations Environment Programme Chemicals, 2002. Regionally based assessment of persistent toxic substances (RBA/PTS): North America regional report. Global Environment Facility.

Van Aken, B., Correa, P.A., Schnoor, J.L., 2010. Phytoremediation of polychlorinated biphenyls: new trends and promises. Environ. Sci. Technol. 44, 2767-2776. 
Wang, S., Zhang, S.Z., Huang, H.L., Christie, P., 2011. Behavior of decabromodipheny ether (BDE-209) in soil: effects of rhizosphere and mycorrhizal colonization of ryegrass roots. Environ. Pollut. 159, 749-753.

Xu, L., Teng, Y., Li, Z.G., Norton, J.M., Luo, Y.M., 2010. Enhanced removal of polychlorinated biphenyls from alfalfa rhizosphere soil in a field study: the impact of a rhizobial inoculum. Sci. Total Environ. 408, 1007-1013.
Zhao, X.R., Qin, Z.F., Yang, Z.Z., Zhao, Q., Zhao, Y.X., Qin, X.F., Zhang, Y.C., Ruan, X.L. Zhang, Y.F., Xu, X.B., 2010. Dual body burdens of polychlorinated biphenyls and polybrominated diphenyl ethers among local residents in an e-waste recycling region in Southeast China. Chemosphere 78, 659-666. 